\title{
Points to consider for laboratories reporting results from diagnostic genomic sequencing
}

\author{
D.F. Vears $\mathbb{1}^{1,2} \cdot$ K. Sénécal ${ }^{3} \cdot$ A.J. Clarke $\mathbb{1}^{4} \cdot$ L. Jackson $^{5} \cdot$ A.M. Laberge ${ }^{6} \cdot$ L. Lovrecic ${ }^{7} \cdot$ A. Piton $\mathbb{1}^{8} \cdot$ \\ K.L.I. Van Gassen ${ }^{9} \cdot$ H.G. Yntema ${ }^{10}$ - B.M. Knoppers ${ }^{3}$ P. Borry ${ }^{1,2}$
}

Received: 4 July 2017 / Accepted: 31 October 2017 / Published online: 28 November 2017

(c) European Society of Human Genetics 2018

\begin{abstract}
Although NGS technologies are well-embedded in the clinical setting for identification of genetic causes of disease, guidelines issued by professional bodies are inconsistent regarding some aspects of reporting results. Most recommendations do not give detailed guidance about whether variants of uncertain significance (VUS) should be reported by laboratory personnel to clinicians, and give conflicting messages regarding whether unsolicited findings (UF) should be reported. There are also differences both in their recommendations regarding whether actively searching for secondary findings (SF) is appropriate, and in the extent to which they address the duty (or lack thereof) to reanalyse variants when new information arises. An interdisciplinary working group considered the current guidelines, their own experiences, and data from a recent qualitative study to develop a set of points to consider for laboratories reporting results from diagnostic NGS. These points to consider fall under six categories: (i) Testing approaches and technologies used, (ii) Approaches for VUS; (iii) Approaches for reporting UF, (iv) Approaches regarding SF; (v) Reanalysis of data \& re-contact; and vi) Minors. While it is unclear whether uniformity in reporting across all laboratories is desirable, we hope these points to consider will be useful to diagnostic laboratories as they develop their processes for making decisions about reporting VUS and UF from NGS in the diagnostic context.
\end{abstract}

\section{Introduction}

Next generation sequencing (NGS) encompasses a range of non-Sanger-based, high-throughput DNA sequencing technologies, including disease-specific targeted capture sequencing, whole exome sequencing (with or without filtering using disease-specific bioinformatic panels), and whole genome sequencing. Although NGS technologies are

D. F. Vears

Danya.Vears@kuleuven.be

1 Center for Biomedical Ethics and Law, Department of Public Health and Primary Care, KU Leuven, Leuven, Belgium

2 Leuven Institute for Human Genetics and Society, Leuven, Belgium

3 Centre of Genomics and Policy, McGill University, Montreal, Canada

4 Division of Cancer \& Genetics, School of Medicine, Cardiff University, Cardiff, Wales, United Kingdom

5 University of Exeter Medical School, University of Exeter, Exeter, United Kingdom well-embedded in the clinical setting for identification of genetic causes of disease, guidelines issued by professional bodies are inconsistent regarding some aspects of reporting results. First, most recommendations do not provide detailed guidance about whether or not variants of uncertain significance (VUS) should be reported by laboratory personnel to clinicians [1-5]. Second, although guidelines generally recommend a targeted approach to sequencing in
6 Department of Pediatrics, Université de Montréal, Medical Genetics, CHU Sainte-Justine; CHU Sainte-Justine Research Center, Montreal, Quebec, Canada

7 Clinical Institute of Medical Genetics, University Medical Center Ljubljana, Ljubljana, Slovenia

8 Molecular diagnostic laboratory, Strasbourg University Hospitals, Strasbourg, France

9 Department of Genetics, University Medical Center Utrecht, Utrecht, The Netherlands

10 Department of Human Genetics, Radboud University Medical Centre, Nijmegen, The Netherlands 
order to limit identification of unsolicited findings (UF) (i.e., variants in disease-causing genes unrelated to the original rationale for testing and identified incidentally during the course of the analysis [3]), these documents give conflicting messages regarding whether UF should be reported if they are identified during the course of the analysis, with recommendations ranging from suggestions that UF may be reported [4], to allowing patients to choose [1], to leaving it to the discretion of the laboratories to decide $[2,3]$. Third, recommendations regarding secondary findings (SF) (i.e., variants in disease-causing genes unrelated to the original rationale for testing and actively sought during the analysis [4]) differ between those issued by ACMG [4, 5] and the European and Canadian bodies [1-3]. Finally, these recommendations vary regarding the extent to which they address the duty (or lack thereof) to re-analyse variants as new information arises and with whom the responsibility to request reanalysis rests [1-3].

A recent qualitative study in which 26 interviews were conducted with 27 laboratory personnel, representing 24 laboratories in Europe (12), Canada (5) and Australasia (7), highlighted that there was considerable variation in practice with regards to the reporting of variants by laboratories following NGS [6]. The participants highlighted that, despite the classification guidelines, variant classification was a real challenge [6]. Regarding reporting practices, some participants indicated that they limit their reporting to variants thought to be related to the phenotype that motivated the test. However, the study found that a large proportion of the laboratories report UF to the referring clinician [6]. Of those that report UF, some report when they consider the variant to be medically actionable, whereas others report based on medical relevance, regardless of actionability. With regards to variant pathogenicity, although some laboratories only report likely pathogenic (class 4) and pathogenic (class 5) variants, the study found that it was more common for laboratories to report VUS (class 3) when they are identified in genes related to the clinical question [6] (manuscript under review). Some laboratories also report VUS in genes not yet associated with the condition. The challenges presented by participants within the study, alongside the lack of consensus across recommendations, highlights a need for more specific guidance for laboratories when reporting results from NGS.

\section{Participants and methods}

A working group of 12 experts was formed in order to develop a set of points to consider for laboratories reporting results from diagnostic NGS. The group included clinical geneticists (4), laboratory specialists (3), ethicists (2), researchers (1) and lawyers (2) from Europe, Canada and
Australia. Each participant was selected based on their individual area of expertise and the composition of the group as a whole. The goal of the working group was to develop suggestions for clinical laboratory scientists and policy makers regarding the reporting of VUS, UF and SF from diagnostic NGS. Two meetings were held to include the perspectives of all the members of the working group: (1) in Montreal, October 2016, and (2) in Paris, December 2016. The working group considered the current guidelines, their own experiences, and data from the qualitative study described above [6]. A preliminary set of points to consider were drafted during the second meeting and circulated iteratively to the working group for refinement.

The points to consider are clustered under six categories: (i) Testing approaches and technologies used, (ii) Approaches for VUS; (iii) Approaches for reporting UF, (iv) Approaches regarding SF; (v) Reanalysis of data \& re-contact; and (vi) Minors. The rationales that underscored the decisions of the working group on these points are provided.

\section{Results and discussion}

\section{Testing approaches and technologies used}

The working group has not issued a formal recommendation regarding the testing approach and the technologies used, because we believe that these decisions require careful consideration and the balancing of a number of different factors for each patient. The working group believes that these decisions should be made at the discretion of each laboratory depending on (a) the clinical question, (b) the age of the patient, (c) the technologies available to them, (d) their resources, including financial and personnel resource restrictions, and (e) the healthcare system under which they are operating.

First, whether a trio-based analysis, as opposed to a proband-only analysis, should be used depends on the clinical question under investigation. For example, if the patient has non-familial intellectual disability, where there are many potentially causative genes and a de novo causative variant is expected, using a trio-based analysis is the best strategy. For other conditions with fewer related genes and where autosomal recessive inheritance is suspected, such as deafness or blindness, proband-only analysis may be appropriate. Second, whether trio-based analysis is possible will depend on the age of the patient, as it is easier to collect samples from the parents of paediatric patients than adults where it is more difficult or, in some cases, impossible. Third, the technology being used by the laboratory also influences this decision. Trio-based analysis is most appropriate when using whole exome/genome sequencing or large panels (such as the Mendeliome), rather than smaller, targeted, disease-based 
panels. Laboratories should also take into account what is requested by the referring clinician.

Finally, financial factors need to be considered in determining the most appropriate testing strategy, such as whether the healthcare system is publicly or privately funded, the financial situation of the patient and their family, and the sequencing budget of the laboratory. Electing a trio-based sequencing strategy might result in fewer probands being sequenced. However, the cost of analysing the exome data, which takes considerably longer for proband-only analysis, and the time required for reanalysis if the parents' samples are collected retrospectively (as well as the time required to recontact them and provide proper information), need to be considered, as does the cost of storage of parental samples.

We suggest that, if the laboratory does not have financial constraints, using a trio-based analysis for exome sequencing in paediatric patients would be the ideal strategy to minimize the number of variants identified. If this strategy is not possible, clinicians should tell parents at the initial appointment that their samples might be required for validation, or to provide additional information regarding the significance of variants identified in the child. This will circumvent the need for an additional appointment with the referring clinician or genetic counsellor. In cases of an urgent nature, it would be best if parental samples were collected at the same time as the proband to hasten a diagnosis. When parental samples are taken, for either confirmatory testing or a trio-based analysis, parents' expectations for identification of causative variants or UF need to be addressed. It should be made clear, during the consent process, that the samples are being used to make sense of the results of the child, and the laboratory will not seek to analyse the parents' sequence data.

\section{Approaches for variants of uncertain significance}

\section{In general, a targeted approach to analysis of data from next generation sequencing should be considered}

This recommendation is in line with those of the CCMG, ESHG and EuroGentest that recommend a targeted approach to analysis of genomic data [1-3]. In order for a targeted approach to be effective, using either targeted gene-capture or exome sequencing with bioinformatic filtering, the laboratory must have a clear idea of the clinical question that is being asked. Therefore, detailed and accurate information about the clinical phenotype of the patient is required to determine which genes should be included in the analysis. Detailed phenotypic information is also crucial for deciding which variants to report. Although laboratories can contact the referring clinician after sequencing takes place to get more details about the patient's phenotype, and how this relates to the variants identified, this additional step can be time consuming and would be unnecessary if more information was provided at the time of the request. We suggest it is not good clinical practice for laboratories to perform exome or large panel sequencing until sufficient clinical information is received from the clinician. An exception would be made for neonatal intensive care unit patients where (a) analysis is timecritical, and (b) the phenotype of the patient might be evolving rapidly.

Of course, there are likely to be cases where, regardless of the level of detail provided at the time of the request, further clarification is required in order to determine whether a variant is causative of the disease phenotype. This may include further clinical or biochemical tests, or additional sample collection from parents/family members to determine segregation patterns. In these cases, laboratory personnel should consider contacting the referring clinician prior to issuing a report or state in the report which complementary tests could be undertaken to determine the causative nature of the variant.

\section{Variants of uncertain significance (VUS) that are identified in known genes, which are related to the clinical question but with insufficient evidence of pathogenicity, should be reported to clinicians}

Despite best efforts, it is not possible to classify some variants as either (likely) benign or (likely) pathogenic. These VUS may be identified (1) in genes related to the clinical question where there is insufficient evidence of pathogenicity, (2) in genes not yet associated with a disease but where the biological functioning is known to some degree [7], or (3) in genes that are known to be diseasecausing but that are unrelated to the clinical question (i.e., a UF of uncertain significance). Current practices in the reporting of VUS are variable. Some laboratories studied are adopting a cautious approach and are not reporting any VUS to clinicians, whereas others are reporting VUS that fall under category (1), or categories (1) and (2) as listed above [20] (manuscript under review).

While the ACMG guidelines explicitly state that they do not support the reporting of VUS, the CCMG recommendations do not explicitly address whether VUS should be reported [1, 5]. However, they do recommend that the analysis should be limited to genes that are known to be disease-causing, regardless of whether they are related to the clinical question [1]. Although the ESHG also does not address whether VUS should be reported [3], EuroGentest leaves reporting of VUS to the discretion of the laboratory [2]. The Dutch best practice guidelines state "only clearly causal variants or very strong candidate variants that suggest/predict functional impairment and warrant further testing in the family, require reporting" [8]. 
The working group discussed the need for a balance between reporting less, which risks missing a diagnosis, and reporting too many VUS, leading to the potential for the mis- and over-diagnosis of patients. As the field progresses, reporting VUS might become more prominent, particularly by commercial laboratories who wish to limit liability. However, as more patients have sequencing performed and the number of variants added to variant databases (such as ClinVar) increases, our ability to classify variants as (likely) benign or (likely) pathogenic will also increase and the list of VUS will eventually diminish.

It was agreed that VUS in the first category (i.e., where the gene is known, relevant to the clinical question, but lacking sufficient evidence of pathogenicity), should be reported. This is because it is a potential answer to the clinical question. As part of this discussion, the working group determined that the decision on whether a single VUS in a gene with an autosomal recessive inheritance pattern should be reported would depend on the level of coverage for that gene (i.e., what the likelihood is that another potentially causative variant has been missed in the analysis) or the known frequency of mutations that are not detectable using that particular technology, and therefore the extent to which the VUS could be part of the answer for the patient. For this reason, patients should not be asked whether they want VUS to be returned. If patients do not want to receive information relevant to their clinical question they should not undergo sequencing.

\section{VUS identified in genes not yet associated with the condition but where there is evidence the variant may be causative of the patient's phenotype, should be reported to clinicians}

The working group agreed that in some cases it is appropriate for VUS that fall into category 2 (i.e., where the gene is not yet associated with the condition but where the biological functioning is known to some degree), to be reported. This decision should depend on what is known about the nature of the gene and if it could potentially cause the phenotype of the patient. When reporting, laboratory specialists should ensure that there is sufficient evidence to suggest both relevance of the gene to the phenotype and variant pathogenicity (e.g., a de novo, truncating mutation suspected to cause a loss of function).

\section{VUS in genes not related to the phenotype of the patient should not be reported, including those in known disease- causing genes unrelated to the clinical question}

This is because these VUS are not relevant to the original rationale and are therefore not potential answers for the patient.
5. When VUS are reported, it should be in a way that distinguishes them from likely pathogenic or pathogenic mutations, such as on a separate page of the report or in a table. Evidence should be provided in the report outlining the reasoning behind their classification

The recent qualitative study identified that some of the laboratory personnel interviewed were concerned that health professionals would over-interpret the significance of VUS if they were reported, which may lead to the incorrect diagnosis of patients and unnecessary follow up [6] (manuscript under review). The working group discussed whether part of the role of the referring clinician is to filter the variants that are reported to them and to decide which of those variants it is appropriate to report to the patient. This is likely to be particularly challenging when the referring specialist does not have specific training in genetics. It is unrealistic to expect laboratories to tailor their reports to the experience of the referring clinician. Therefore, the report should clearly distinguish between VUS and (likely) pathogenic variants. Some participants interviewed explained that they have found that having either a separate page of the report, or a separate table, with the VUS has helped reduce the confusion for clinicians.

In addition, it is important to include sufficient details about the lines of evidence which have led to the classification of the variant as a VUS, rather than being (likely) benign or (likely) pathogenic. This should include whether the variant has been associated with a disease in the literature, whether it is in a variant database, such as ClinVar or the Human Gene Mutation database, the type of mutation (i.e., truncating, de novo, loss of function etc.) and the likely impact of this on the protein.

6. Any reported VUS, along with phenotypic data, should be shared in a relevant database, such as ClinVar, to assist the diagnosis of other patients

The sharing of genomic data in databases, such as ClinVar, is important for the accurate interpretation of results from NGS. Access to large datasets of variants allows for comparisons between patient phenotypes and genotypes, assisting classification of variants. For this reason, data sharing is beneficial for the individual patient, other patients with the same condition, and the genetic community more broadly (including researchers and clinicians) and should be promoted [9]. However, data sharing can be ethically challenging, particularly with regards to issues of privacy and confidentiality [10]. Although data may be anonymised or deidentified, the nature of genomic information means that it is potentially reidentifiable, particularly in patients with rare diseases. In these cases, linking phenotypic data with genomic data is crucial for determining whether a variant is causative. However, this makes these patients 
more easily identifiable. We suggest that reported variants should be routinely shared in mutation databases provided the patient is deidentified, appropriate data protection/privacy mechanisms are in place, and patients are notified their data will be shared.

\section{Approaches for reporting unsolicited findings}

In this section we are referring to the reporting of UF in competent, adult patients—reporting in minors is addressed in a separate section. By UF, we are referring to known pathogenic variants in disease-causing genes that are unrelated to the original rationale for testing and that are identified inadvertently. These variants have also been referred to as incidental or unanticipated findings [11,12]. We distinguish these from SF, which we discuss in the following section. While the existing guidelines by CCMG, EuroGentest, and ESHG [1-3] recommend targeting data analysis in order to limit the identification of UF, recommendations on whether UF should be reported by laboratories to clinicians if they are identified vary. While the ACMG does not explicitly recommend reporting of unsolicited findings [4,5], the CCMG suggests that competent adults should be given the option to choose to receive UF [1]. The ESHG and EuroGentest leave the decision as to whether UF are reported to the laboratory, but advocate for a defined protocol $[2,3]$. Yet the ESHG does suggest that if a UF is suggestive of a serious, but treatable or preventable, health issue, this should be reported to the clinician so it can be relayed to the patient [3]. The working group has attempted to find a balance between focusing on the health of patients (and their family members) while respecting patient autonomy.

\section{Laboratories should not report unsolicited findings where there are no health implications for the patient, or their family}

8. Unsolicited findings that are relevant to the health of patients should be reported to clinicians, provided that informed consent has been obtained for such reporting from patients prior to sequencing. A patient's choice not to know unsolicited findings should generally be respected

Of note is that discussions on whether variants that are extraneous to the clinical question should be reported often focus on concepts of 'actionability', 'medical relevance', 'clinical significance' or 'clinical utility' [1, 3, 5, 8, 13]. Yet, these terms are problematic in practice. First, terms such as 'actionability' are very subjective and often based on clinical judgment. Definitions of these terms will vary according to context and individual values, meaning that these terms are likely to be interpreted differently by different people. Does 'actionability' only refer to variants where the knowledge will lead to a particular treatment or intervention for the patient?
Or does it also incorporate potential interventions for family members? While some may not consider identification of carrier status in a child as actionable or of clinical utility, because the information will not change the child's clinical management, others may consider it to have clinical utility because the parents can use this information to inform their own future reproductive decisions. These terms are also time dependent. What might not be actionable now may become actionable in the future. For these reasons we suggest that UF that are relevant to the health of patients, regardless of actionability, should be reported, provided the competent, adult patient has opted-in to receive these findings. This would also include UF with low or incomplete penetrance, provided the variant was (likely) pathogenic.

Patients should be asked whether they wish to receive UF prior to testing, at the time consent is being obtained for the test. If patients have opted out of receiving UF, their right not to know should generally be respected, although we acknowledge there may be some specific circumstances where this may not be appropriate $[14,15]$. We acknowledge that the right not to know is not absolute; the duty to provide information may supersede this. To ensure that the decision not to receive UF is informed, the consent process should involve adequate genetic counselling in which the types of UF which may be identified are explained, as well as the likelihood that this will occur based on the analysis being performed. In order to respect a patient's right not to know UF, laboratories should have protocols, such as filtering strategies, to prevent identification of UF (as much as is feasible) so they are not placed in a position where they identify UF that they do not report. We acknowledge that allowing patients to refuse receiving UF removes the opportunity for other family members to potentially benefit from the knowledge of these UF. However, it is important to keep in mind that the primary reason for genomic sequencing in this situation is to identify the cause of the genetic disease in the patient, not for cascade or population screening.

\section{If carrier status is identified in adults, regardless of whether it relates to the clinical question, it should be reported if informed consent is obtained prior to testing. This is because knowing one's carrier status can increase reproductive options}

Based on the above rationale, carrier status for autosomal (e.g., cystic fibrosis) and X-linked recessive conditions (e.g., Duchenne Muscular dystrophy) should be reported, but only when this is identified inadvertently. Laboratories should not analyse these genes systematically and it should be made clear to patients that filtering strategies used by the laboratory may minimise the chance of identifying carrier status. If a laboratory chooses to actively search for carrier status, this should be performed separately from the original 
analysis and only if prior informed consent for the separate analysis has been obtained from the patient.

\section{Reporting unsolicited findings where there is} insufficient evidence of pathogenicity (i.e., VUS) should be avoided

This point is based on the argument that only variants that are relevant to the health of patients should be reported. In addition, although (clearly) all variants should be analysed carefully before they are reported, UF should be analysed even more carefully than variants related to the phenotype of the patient because we do not have clinical data to confirm the pathogenicity of the variants.

\section{Approaches regarding secondary findings}

By SF we refer to variants in disease-causing genes that are actively searched for during the analysis but that are not relevant to the clinical question. The most well known guidelines that address the reporting of SF are those by the ACMG which have proposed a list of 59 (previously 56) variants that they recommend should routinely be reported to clinicians following NGS [4, 5]. While ESHG and EuroGentest do not explicitly address this issue, their recommendation for a targeted approach to limit the identification of UF implies that they do not support actively searching for SF $[2,3]$. The CCMG explicitly states they '[...]do not endorse the intentional clinical analysis of disease genes unrelated to the primary indication, even if the results might be medically actionable[...]' [1].

\section{Laboratories should not actively search for secondary findings. However, if a laboratory chooses to actively search for secondary findings, this should be performed separately from the original analysis and only if prior informed consent for the separate analysis has been obtained from the patient}

If a variant on the ACMG list is identified as a UF then it should be reported. However, the working group agreed that not only is there no obligation for laboratories to actively search for SF, based on our current knowledge, but that it is best practice not to perform this type of analysis for SF. This is because (1) it is not relevant to the primary question, and (2) this constitutes opportunistic screening and if population screening is to be implemented, it should fulfill the WHO or related criteria for screening [16].

If laboratories choose to offer this as a service to competent, adult patients, it is important that the patients are asked to consent to this as an additional, secondary analysis. In order to ensure that their decision whether to receive SF is as informed as possible, the consent should be 'opt-in' and accompanied by adequate genetic counselling. The laboratory should undertake the necessary validation procedures with the assistance of specialists in the field to ensure the pathogenicity of the variants being screened is as certain as possible at the time of the analysis. Results of this analysis should be reported on a separate page of the report to distinguish them from results related to the clinical question.

\section{Reanalysis of the data \& re-contact}

\section{There is no obligation for laboratories to routinely} reanalyse data. However, if the laboratory learns that the status of a specific variant has been reclassified from a pathogenic or likely pathogenic to a benign or likely benign variant, or vice versa, it is good clinical practice for laboratories to identify patients with this variant from their database and issue a new report to the referring clinician. Any other requests for reanalysis of sequence data should be initiated by the patient, either via the referring clinician, or another clinician who has taken over their care

The working group agrees with statements by the CCMG and EuroGentest that there is no duty for laboratories to reanalyse sequence data in light of additions to variant databases [1,2]. The service the laboratory provides only includes the initial analysis, not subsequent reanalysis of the data. The report issued by the laboratory should be clear in stating that the classification of VUS is based on the knowledge at the time the analysis takes place. This is distinct from any reanalysis that is performed by laboratories in order to increase the quality of the sequence data. We suggest that any requests for reanalysis to take place should be initiated by the patient (or their parents) via their referring clinician, rather than by the laboratory or by the clinician independently. This is to ensure the patient still requires a genetic diagnosis and to avoid situations where sequence data has been reanalysed and the patient or their family is lost to follow up. However, since there is the potential for social disadvantage to increase inequity by distorting the provision of health care in this regard [17], clinical services may want to issue reminders to patients via their patient databases to prompt re-contact.

There may be situations where new evidence becomes available for a particular variant that results in its reclassification. This could be from benign, likely benign, or VUS, to pathogenic or likely pathogenic, in which case the variant becomes the answer for the patient. The reclassification could also be from pathogenic or likely pathogenic to benign, likely benign, or VUS, in which case the variant is no longer considered to be the cause of the patient's disease. Both are important as they may change patient management and have implications for the broader family, such as influencing surveillance or reproductive decisions. This, of course, only applies to variants either in genes known to be related to the 
clinical phenotype or in genes not yet associated with the condition but where causation might be plausible, not variants in disease-causing genes that are unrelated to the phenotype (i.e., UF). In this situation, the analysis of a variant identified for a particular patient (or patients) that carries that variant are being reinterpreted, rather than reanalysed. If this occurs, it is good clinical practice for the laboratory to reissue a report to the referring clinician so they can attempt to communicate this information to the patient.

\section{Minors}

Current guidelines are discordant in their stance on the reporting of results from NGS in children. While the ACMG suggests that the list of SF should be reported, both in adults and children, the CCMG makes a clear distinction in the reporting practices of UF between children and adults, advocating that ' $[\ldots]$ incidental results that reveal risk for a highly penetrant condition that is medically actionable during childhood should be reported to the parents' [1,4]. Although EuroGentest does not specifically address this issue, ESHG highlights a need to establish guidelines around the disclosure of UF in minors in light of the ethical tension between protecting the future autonomy of the child and parents' rights to make decisions for their family $[2,3]$.

13. In children, unsolicited findings that are (a) relevant to their health during childhood or adolescence, and (b) medically actionable, should be reported to parents

The recommendation to report variants that are medically actionable and that are likely to present in childhood or adolescence is in agreement with that of the CCMG [1]. The working group decided this was appropriate because reporting these variants can lead to treatments or preventative strategies that can promote the child's health and is therefore in line with the best interests of the child [18].

\section{Unsolicited findings in children that identify a risk for} adult-onset diseases should be returned in circumstances where the UF has significant implications for the health of a family member, or the child, during their lifespan. The decision to report should consider the probability of the risk occurring, whether the condition is life-threatening, and if treatment or an effective method of prevention is available. This should be determined on a case-by-case basis, and with the informed consent of the parents prior to testing

The rationale behind this is that it is not good practice for laboratories to have identified UF with significant health implications and not report them, particularly when there is no system in place to report the findings when the child reaches adulthood. However, UF that indicate a risk for an adult onset condition where there are no actions that can be implemented to treat or prevent the onset of the condition in either the child, or a parent or family member, should not be reported. Reporting should only take place where the parents have been informed of this possibility and have elected to receive these UF. It must be made clear to the parents which results will or will not be disclosed and, if the laboratory chooses not to report the finding, that the patient can contact the laboratory when they reach adulthood. There is no obligation for either the laboratory or the clinician to re-contact the patient when he/she reaches adulthood if the results have not been communicated. If parents' status for such variants is unknown (i.e., the mutation was not identified using a triobased approach), provided parents have elected to receive UF during the consent process, the mutation should be reported. This is because it is very likely that one of the parents carries the mutation and disclosure of the mutation may therefore benefit the health of the parents. Finally, in children in whom it is clear they will never become competent, UF for late onset conditions should be reported and disclosed to the parents, provided they have elected to receive these findings.

\section{If carrier status for any condition is identified in children, it should be reported if informed consent has been obtained from the parents prior to testing. This is because knowing one's carrier status can increase reproductive options}

Carrier status should be reported because it provides potentially actionable information to the parents to make health-related decisions concerning potential future children. This reporting should only take place where the parents have been informed of this possibility and have elected to receive this information.

16. Opportunistic screening for secondary findings should not be performed in children, regardless of whether the parents desire this information

As per the rationale for not performing this 'opportunistic screening' in adults, it is not relevant to the primary question and, if screening is to be implemented it should fulfill the criteria for population screening. In addition, this is equivalent to performing predictive testing in children for adult onset conditions, yet without a positive family history to assist the child to make sense of their genetic risks.

\section{Conclusion}

While it is unclear whether uniformity in reporting across all laboratories is desirable, in this document we have attempted to provide laboratories with clear points to consider on how to 
report VUS and UF from NGS in the diagnostic context. Of course, these suggestions are appropriate at this point in time and will require further consideration as the field progresses. In addition, new data protection regulations in the European Union mean that, as of 25 May 2018, data subjects will have the right to access their own personal data as held by the data controller (i.e., a third party) [19]. This change constitutes a dramatic shift in data transparency and empowerment of data subjects regarding their personal data and may, in the future, have an impact on laboratory reporting practices.

Acknowledgements We acknowledge the support of the Research Fund Flanders (Belgium) and the Ministère de l'Économie, de la Science et de l'Innovation du Québec, PSR-SIIRI-850 (Canada). We also thank Prof François Rousseau, Université Laval, Quebec for his participation in the working group.

\section{Compliance with ethical standards}

Conflict of interest The authors declare that they have no competing interests.

\section{References}

1. Boycott K, Hartley T, Adam S, et al The clinical application of genome-wide sequencing for monogenic diseases in Canada: Position Statement of the Canadian College of Medical Geneticists. J Med Genet 2015;52:431-7.

2. Matthijs G, Souche E, Alders M, et al Guidelines for diagnostic next-generation sequencing. Eur $\mathrm{J}$ Hum Genet 2016;24:1515.

3. van El CG, Cornel MC, Borry P, et al Whole-genome sequencing in health care: Recommendations of the European Society of Human Genetics. Eur J Hum Genet 2013;21:S1-S5.

4. Kalia SS, Adelman K, Bale SJ, et al Recommendations for reporting of secondary findings in clinical exome and genome sequencing, 2016 update (ACMG SFv2.0): a policy statement of the American College of Medical Genetics and Genomics. Genet Med 2016;19:249-55.

5. Green RC, Berg JS, Grody MWW, et al ACMG recommendations for reporting of incidental findings in clinical exome and genome sequencing. Genet Med 2013;15:565-74.

6. Vears DF, Sénécal K, Borry P. Reporting practices for unsolicited and secondary findings from next generation sequencing technologies: Perspectives of laboratory personnel. Hum Mutat 2017;38:905-11.
7. Richards S, Aziz N, Bale S, Bick D, Das S, Gastier-Foster J. Standards and guidelines for the interpretation of sequence variants: A joint consensus recommendation of the American College of Medical Genetics and Genomics and the Association for Molecular Pathology. Genet Med 2015;17:405-24.

8. Weiss MM, Van der Zwaag B, Jongbloed J, et al Best practice guidelines for the use of next-generation sequencing applications in genome diagnostics: A national collaborative study of Dutch genome diagnostic laboratories. Hum Mutat 2013;34:1313-21.

9. Lu JT, Campeau PM, Lee BH. Genotype-phenotype correlationpromiscuity in the era of next generation sequencing. New Engl $\mathrm{J}$ Med 2014;371:591-3.

10. Fiore RN, Goodman KW. Precision medicine ethics: selected issues and developments in next-generation sequencing, clinical oncology, and ethics. Curr Opin Oncol 2016;28:83-87.

11. Christenhusz GM, Devriendt K, Dierickx K. Secondary variants-in defense of a more fitting term in the incidental findings debate. Eur J Hum Genet 2013;21:1331-4.

12. Tan N, Amendola LM, O'Daniel JM, et al Is "incidental finding" the best term?: a study of patients' preferences. Genet Med 2017;19:176-81.

13. Dheensa S, Shkedi-Rafid S, Crawford G, Bertier G, Schonstein L, Lucassen A: Mangement of incidental findings in clinical genomic sequencing: eLS. Chichester: John Wiley \& Sons Ltd., 2016.

14. European Council: Convention for the Protection of Human Rights and Dignity of the Human Being with regard to the Application of Biology and Medicine: Convention on Human Rights and Biomedicine. Oviedo: 1997.

15. World Medical Association: Declaration of Lisbon on the Rights of the Patient. Adopted by the 34th World Medical Assembly, Lisbon, Portugal, and reaffirmed by the 200th WMA Council Session, Oslo, Norway, 2015]: 1981 [amended by the 47th WMA General Assembly, Bali, Indonesia, 1995; editorially revised by the 171st WMA Council Session, Santiago, Chile, 2005.

16. Wilson J, Jungner G: Principles and practice of screening for disease. Geneva: World Health Organization, 1968.

17. Hart JT. The inverse care law. Lancet 1971;297:405-12.

18. Sénécal K, Vears DF, Bertier G, Knoppers BM, Borry P. Genomebased newborn screening: a conceptual analysis of the best interests of the child standard. Personal Med 2015;12:439-41.

19. European Union: Regulation (EU) 2016/679 of the European Parliament and of the Council of 27 April 2016 on the protection of natural persons with regard to the processing of personal data and on the free movement of such data, and repealing Directive 95/46/EC (General Data Protection Regulation). 2016.

20. Danya F. Vears, Karine Sénécal, Pascal Borry, (2017) Reporting practices for variants of uncertain significance from next generation sequencing technologies. European Journal of Medical Genetics 60 (10):553-558 